\title{
Live E! Project: Sensing the Earth
}

\author{
Masaya Nakayama ${ }^{1}$, Satoshi Matsuura ${ }^{2}$, \\ Hiroshi Esaki ${ }^{1}$, and Hideki Sunahara ${ }^{2}$ \\ 1 The University of Tokyo, \\ 7-3-1 Hongo, Bunkyo-ku, Tokyo, 113-8656, Japan \\ ${ }^{2}$ Nara Institute of Science and Technology, \\ 8916-5 Takayama-cho, Ikoma-shi, Nara, 630-0192, Japan \\ nakayama@nc.u-tokyo.ac.jp, sato-mat@is.naist.jp, \\ \{hiroshi, suna\}@wide.ad.jp \\ http://www.live-e.org
}

\begin{abstract}
The Live E! project is an open research consortium among industry and academia to explore the platform to share the digital information related with the earth and our living environment. We have getting a lot of low cost sensor nodes with Internet connectivity. The deployment of broadband and ubiquitous networks will enable autonomous and global digital information sharing over the globe. In this paper, we describe the technical and operational overview of Live E! project, while discussing the objective, such as education, disaster protection/reduction/recovery or business cases, and goal of this project activity.
\end{abstract}

Keywords: Live E!, sensor network, environmental information.

\section{Introduction}

Recent natural disaster, e.g., hurricane or global warming, or heat island effect in the metropolitan let increase the attention and interesting on grasping the detailed status of space. This is because, due to these disasters, our social life and business activity could be seriously degraded or sometimes be damaged. When we could realize the relationship of cause and effect, it would be expected to reduce and protect the damage by these disasters. The structure change of metropolitans in the developed countries and rapid inflation of cities in Asian countries make complex and difficult to realize the real status and tendency of global weather system. As symbolized by Kyoto Protocol proposed by United Nation in February of 2005, so called COP3, it is realized as the urgent and serious global agenda to reduce or to stop the global warming effect.

We have getting a lot of low cost sensor nodes with Internet connectivity. The deployment of broadband and ubiquitous networks will enable autonomous and global digital information sharing over the globe, using these sensor nodes. When these wide variety of sensor nodes are autonomously connected and the sensor information let available to all the node on the Internet space, different types of sensor information, e.g., video or still image captured by Web camera, temperature, location, IR-image or chemical, can be integrated for data analysis. Then, 
we will be able to create so wide variety of applications and possibilities. When these sensor nodes are connected with broadband Internet, these information can be available even in real-time fashion. We have realized and proposed the activity, called Live E! Project (http://www.live-e.org), that is a sensor networks sharing all the digital information related with the at large status of the Earth for any purpose and for anyone. We expect that these digital information will be used for various purposes, e.g., educational material, public service, business cases, by the deployment of effective and safe physical space for all the human being.

Internet has been originally invented and developed to use and to share the expensive high performance computers, remotely. In these days, the jobs executed at the computer were numerical calculation for particular work to provide more effective working environment for researchers. Digital information has the following five primitives; generation, collection, circulation, processing, sharing. Through these five primitives, the computer system can improve and innovate the life-style of human being or the professional/commercial activities. Also, we have realized that the ICT can contribute to improve and innovate the human life and industrial activities more effective and safer.

In this paper, we describes the technical and operational overview of Live $\mathrm{E}$ ! project, while discussing it's objective, such as education, disaster protection/reduction/recovery or business cases, and goal of this project activity.

\section{Internet for Facility Networks}

\subsection{Challenges of Internet}

"Internet", not "The Internet", is a logical architecture, that enable autonomous circulation and sharing of digital information. The Internet should provide a transparent platform with the following characteristics.

(1) can use any data-link

(2) can use any data distribution channel

(3) can be used by anyone

This chrematistics (or could be said requirement) are truly critical for ubiquitous networks. In the ubiquitous networks, there is huge number of device nodes (such as sensor nodes), and it is desired all the digital information generated by these device nodes should be available from any node on the Internet space and be able to be shared among all the nodes on the Internet space.

The Internet is the system where the computers are interconnected via any type of data-link, so that the digital data is autonomously transferred from any node to any other node on the Internet space, transparently. Any node on the Internet space should be able to use and process these data, autonomously. In other words, the Internet is;

(a) independent from application

(b) independent from user

(c) independent from data transmission infrastructure 
This means that the Internet satisfy the requirements of "Commons" discussed in [1. The Internet should not have any exclusiveness related with the information exchange.

Internet has experienced major three innovations in the past, and is now exploring the fourth. Past innovations are open global network with TCP/IP, easier use with WWW and professional application with Web Service. The fourth innovation is broadband and ubiquitous. Broadband includes always-on environment, and the ubiquitous means the Internet accommodates embedded devices, such as sensor nodes or actuator nodes.

The facility networks is a kind of embedded network for some particular purpose or for some particular application, while having a strong interaction with the real and physical objects. Since the facility network has a lot of interaction with physical objects, it is said that the network must be dependable. People starts to realize that, in order to build dependable facility network, the network should be operated autonomously and with multi-vendor environment (i.e., open system). Open and autonomous system can provide the alternatives for users. Providing the alternatives to the users leads a competition among vendors for technology improvement and cost reduction, and leads the service continuation capability as a result.

Some of the challenges of the Internet discussed in this paper is (1) how to accommodate wide variety of and large number of sensor/actuator nodes into the Internet space, and (2) identify new applications using the weather sensor nodes deployed in the Live E! project.

\subsection{Facility Networking with TCP/IP}

Facility networking includes factory automation, building automation or home automation, which is the integration of different type of networks, such as sensor network, control network or security network. Especially the role of sensor networking and actuator networking has the important role for the near future facility networking architecture. The facility networking would be toward the integration of real-space and cyber-space using common IP technology.

We have worked with the industry so that the TCP/IP technology, especially the IPv6, is applied to the basic protocol to be shared by various systems in facility networking. Since they have used proprietary technologies for each company and for each sub-systems, they are interested in the IP-Centric system, to achieve highly cost-effective system operation. We had the world first interoperability testing among building automation components with IPv6 transport, using the BACnet [2] and LonWorks [3], in 2004, as shown in figure 1] And, in 2006 , we have achieved the world first interoperability of management and control of system components across these different systems using the Web service over IPv6 infrastructure.

With applying the open technology such as TCP/IP, the system components can be easily replaceable, while achieving the remote monitoring and controlling. For the business operation of "facility", the cost of human labor due to maintenance and the cost of component replacement, modification, improvement and 


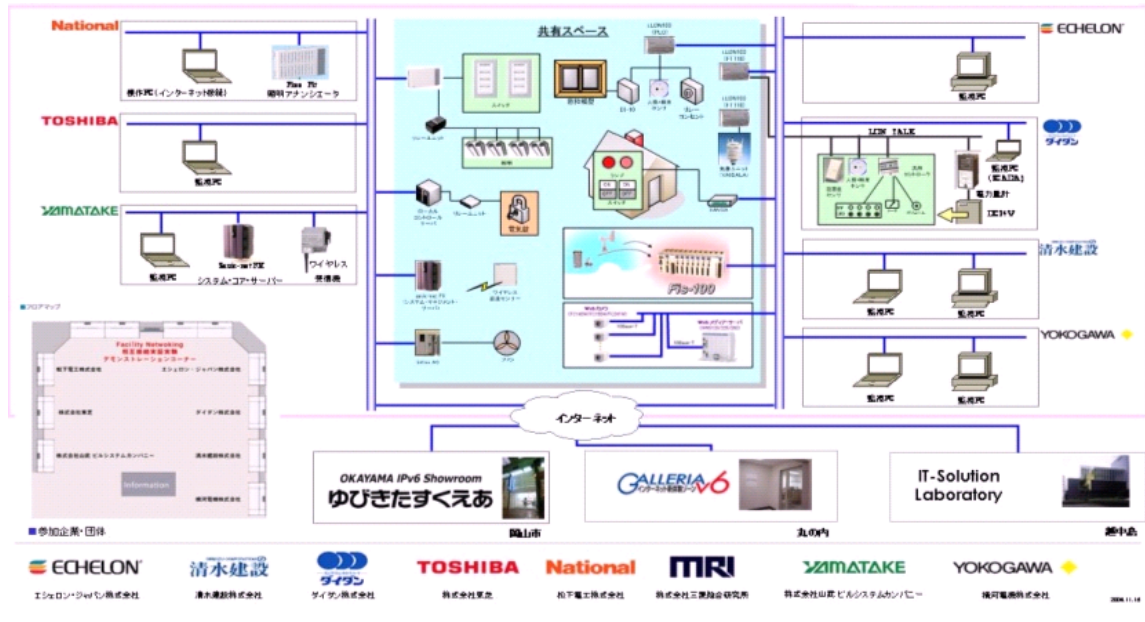

Fig. 1. Interoperability Test in 2004

upgrade occupy a large portion in their financial portfolio. The remote monitoring and control capability by the TCP/IP contributes to the reduction of required human labors, and the multi-vendor environment due to the open networking with the TCP/IP technology contributes to the total cost reduction of system components management by the availability of alternative products. Based on these observation, we have realized that the facility networking industry will be able to enjoy the technical and economical benefits by the adoption of TCP/IP technology.

When we consider the application of and role of sensor networking in the facility networking, there are a lot of potential roles for their effective operation and management. Let consider the application of energy saving in the building automation. In general, the energy cost in the operation of building system (and in the at large facility networks) is extremely large. Especially in Japan, the energy cost is about $1 / 3$, regarding all the life-time cost of building system, that includes the initial designing building system, construction and daily maintenance cost. As well as the benefit of energy saving activity from the view point of economical portfolio for the land-lords, we have another reason why we must perform the energy saving in the facility networking. In 1997, the United Nation has adopted the international energy saving program, so called Kyoto Protocol in COP3 [4]. This is global agenda to preserve the healthy earth by the improvement of global worming effect. One of the important agenda of this program is to reduce the energy consumption by every single office or factory. For example, in Japan, the energy saving program has been applied to all the large scale facility, that has more than 2,000 square-meters footprint. These facilities must reduce the energy consumption $10 \%-20 \%$, as a government regulation.

We have got some private reports from some private companies. They try to energy saving at their computer room, using the temperature sensors, to success 


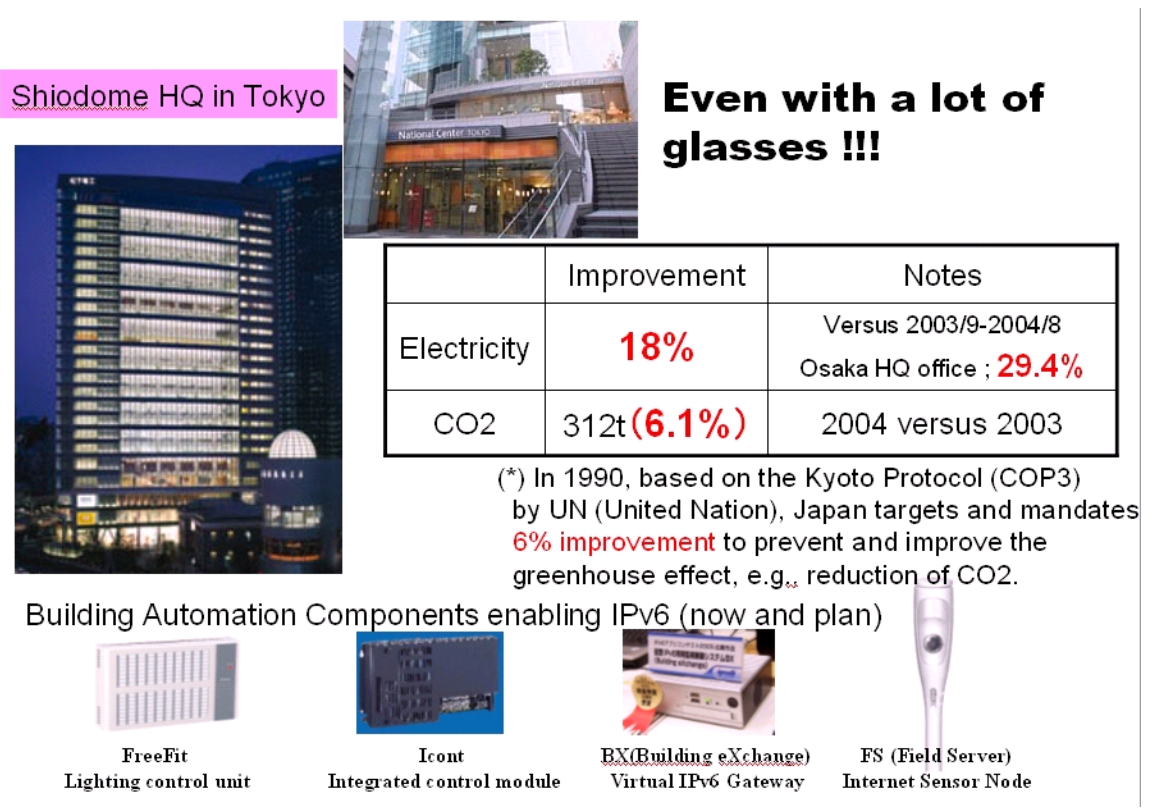

Fig. 2. Energy saving at $\mathrm{HQ}$ of Matsushita Works

reducing about $40 \%$ electricity. Also, at the 24 th floor high recent building system with a lot of glasses, it is reported that $18 \%$ reduction of electricity and $6.1 \%$ reduction of CO2 consumption has been achieved, using sensors and actuators, which are interconnected with open networking protocol, such as TCP/IP. This is shown in figure 2 .

As discussed above, the sensor and actuator networks are not separated or isolated network, anymore. They are interconnected to each other, and they are going to be networked with the usual information network and with the Internet, in order to achieve more cost effective and functional rich system operation, as well as to generate new function or services. The larger sharing of sensor information among various facility networks leads to better and more cost effective and attractive system operation.

\section{Live E! Project}

\subsection{Overview of Live E! Project}

Live E! Project [5] is a R\&D consortium founded by WIDE project and IPv6 Promotion Council, Japan. This project is aiming to establish the platform to share all digital information, generated by sensor devices all over the world. Now, these devices are installed and operated individually by each organization. The information should be related with the live environmental information of the Earth. By sharing this digital information, we will be able to create new 


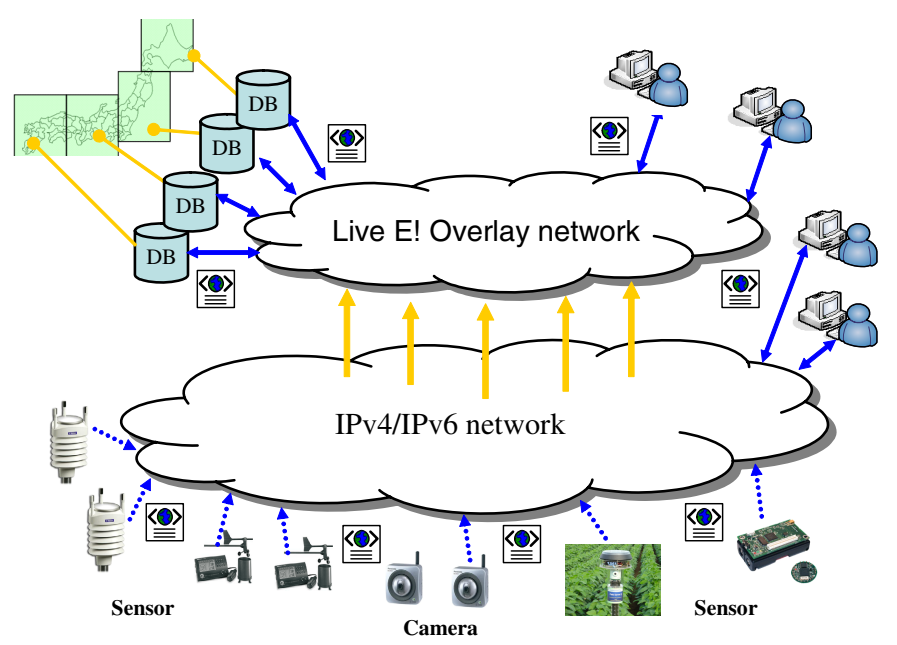

Fig. 3. Overview architecture of Live E! project

applications, which can contribute to safety and effective space (environment). Some applications, such as basic information for the protection of environment (e.g., a heat-island phenomena in metropolitan), educational material, public service, public safety or business applications, could use the common digital information for different purposes. Figure 3 shows the overview architecture of Live E! project. The followings are the working agenda of Live E! project.

(1) By the installation of larger number of digital sensor nodes, the environmental information can be richer and finer, i.e, connectivity is own rewards.

(2) Every individual and every organization should recognize all has the responsibility on the preservation and improvement of global environment and should think all must contribute.

(3) We could give away the ownership on the digital (raw) data for the public, and let them available for all the people on the Internet or on the globe.

(4) Educate and encourage the interesting on the science and technology through the participation on the program and using the data for educational program.

\subsection{Live E! Deployment with Digital Weather Stations}

The related original work would be back in 2001, when the WIDE project with Yokogawa Electric Corp.(http://www.yokogawa.com/) had developed a temperature node with IPv6 protocol stack, called i-node (http://www.i-node.co.jp/).

We have realized that the weather station with Internet connectivity has the following three application areas with single device.

(1) For Educational Material

Weather information data is useful data for education and for research on geophysics. There are wide variety of educational program on geophysics, 


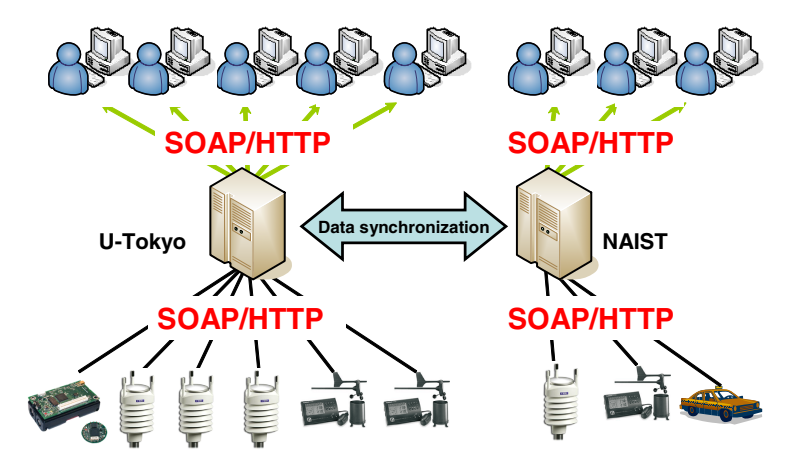

Fig. 4. integrate interface by SOAP

related with the weather system, from the elementary school to the college. Actually, in Live E! project, we have worked on the educational program in the elementary school in Minato-ku in Metropolitan Tokyo, and on the engineering program in some high schools collaborating with university in Hiroshima.

(2) For Public Service, e.g., disaster reduction/recovery

Weather information is very important and critical information for the case of disaster. In these days, we have a lot of natural disasters, such as flooding or earth-quake. Grasping the detailed information for the disaster case is useful for proactive and reactive program. These are disaster protection, reduction, and recovery. For example, the detailed weather information on the road or at the evacuation sites, the people could take appropriate evacuation path. Also, none knows the exact and detailed data on the heat-island effect at metropolitan. We must grasp the real status of town with large number of weather sensors.

In the fiscal year of 2006, Live E! project will deploy the Internet weather sensors for public service at Minato-ku in metropolitan Tokyo 6 and at Kurashiki-city in Okayama. Kurashiki-city [7] is focusing on the disaster protection and reduction, against the flooding due to heavy rain. Minato-ku is focusing on the disaster protection and reduction against some natural disasters (e.g., flooding or earth-quake), and focusing on the understanding the detail of heat-island effect of metropolitan Tokyo.

(3) For Business case

There are a lot of potential business applications, by the use of weather information. One of example would be effective taxi dispatching using the rainfall information. Dispatching the taxi cabs around the area, getting the rain, leads to higher income by the increase of customer. The other example would be electricity power company. Once they can operate total energy control and management system, they could reduce the amount of investments on the power generator or power supplying system, which is very expensive facility for them. Seriously, they may start to think about total portfolio for business investments. 
Table 1. Normalized name \& unit

\begin{tabular}{c|c}
\hline name & unit \\
\hline Temperature & ${ }^{\circ} \mathrm{C}$ \\
Humidity & $\%$ \\
Pressure & $\mathrm{hPa}$ \\
RainFall & $\mathrm{mm} / \mathrm{h}$ \\
WindSpeed & $\mathrm{m} / \mathrm{s}$ \\
WindDir & $\circ$ \\
\hline
\end{tabular}

Table 2. Examples of profile data

\begin{tabular}{c|c}
\hline name & explanation \\
\hline address & address of sensor \\
location & name of installation location \\
ipAddr & IPv4 address \\
ip6Addr & IPv6 address \\
latitude & latitude of sensor \\
longitude & longitude of sensor \\
altitude & altitude of sensor \\
\hline
\end{tabular}

Table 3. examples of SOAP web-services

\begin{tabular}{l}
\hline \hline getCurrentDataAll () \\
- get current data from all sensors \\
\hline getCurrentDataByAreaRect $(\mathrm{x} 1, \mathrm{y} 1, \mathrm{x} 2, \mathrm{y} 2)$ \\
- get data generated in paticular region \\
\hline getDataByTimespan( sensorID, startTime, endTime ) \\
- get data by time-span \\
getCurrentDataByType( sensorType ) \\
- get current data of specific sensor type \\
getProfileAll() \\
- get all profile data \\
getProfileByType( sensorType ) \\
- get profile data of specific sensor type \\
putData( xmlDocument ) \\
- put sensing data \\
setProfile( xmlDocument ) \\
- set profile data
\end{tabular}

\subsection{Data Normalization and Access Method}

In last section, we show the effectiveness of facility networking with TCP/IP. The reason why the facility network can improve energy conservation is that we access to all building information seamlessly on this network. In order to expand this success into the sensor network, first we need to normalize a data format and access method. In this sub-section we introduce our data format with some examples.

The weather information is expressed by XML and transferred among the servers and clients using the SOAP. The sensor is not defined by node, but is defined by each sensor function. A typical internet weather station has multiple sensors in a single station in it, which sensors are temperature, humidity, pressure, rainfall, wind-speed and wind-direction. This typical weather station has 5 objectives defined by XML. Each objective has their own profile information, such as location, sensor type, IP address or name.

As shown in Table 1, we basically adopt MKS-system on our platform. If a new kind of sensor is installed, project member will define its name and the unit (recently, we installed CO2 sensors, illumination sensors, acceleration sensors 
and other sensors). By SOAP web-services, we create a user interface (Figure 4). When user program provides the name of sensor function to web-service, it can access all of sensing data without any conversion. As whole databases are synchronized between two locaitons, users can select web services which they want to use.

As the table 2 shows, we also provide attribute information of sensors called "profile data". We normalize and provide sensing data and profile data as XML document. Application creators freely combine sensing data with profile data to meet each requirement.

Example web service we prepared are shown in Table 3 Here, we introduce an example way to retrieve sensor data. The most popular web-service is "getCurrentDataAll()". By this function, user gets current data generated by all sensors. Following Perl script is an example code to do it.Of course, any language and operating system can access to sensor data by using SOAP.

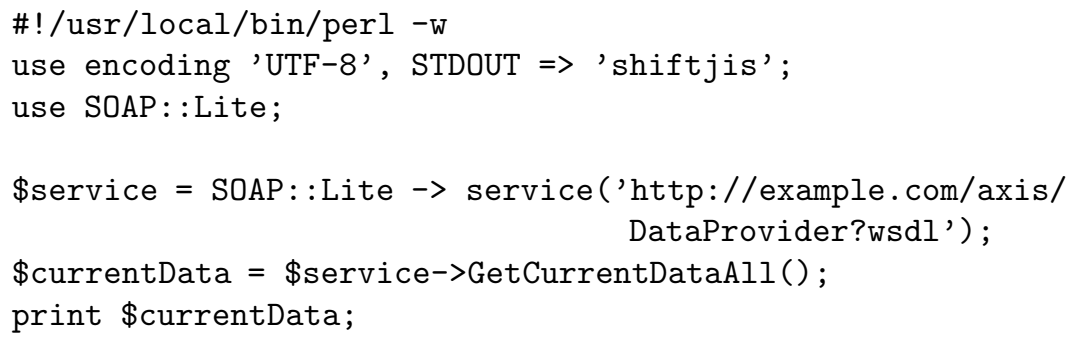

Figure 5 shows the result XML documnets when above example Perl script executed. We standardized XML format. Using other SOAP web-services, user always receives data as same XML format. Defining the name of web-services and XML format contributes to create application easily. In addition, as the above XML documents shows, we define a sensor-group. Sensor-group organizes individual sensors. In above XML document, class of sensor-group is "combinedSensor". This sensor-group organizes a multiple sensor as one of sensors. We define a new class of sensor-group for several purposes. For example, class of "zipCode" is to manage a particular area, and class of "highAccuracy" is to pick up high performance sensors. Of course, we create new web-services by combining several classes. Sensor-group improves a creativeness of raw sensing data.

Here, we introduce some of our applications created by SOAP web-services. Figure 6 shows the example of data displaying. On the web site, user can search each place for detailed data, which are graph of sensors, XML (or CVS) data and pictures of web cameras.

We also provide educational materials. We made a weather information viewer called weather checker (Figure 7). This viewer displays real time data of each place. Students of elementary school constantly check and record the data of this viewer. Students will plot a graph with these data and discuss the difference of daily weather and local climate, and compare climate of home town with other place. Students will take interest in science with such actual experiences. 
<sensorGroup address="東京都文京区弥生2-11-16 " class="combinedSensor" id="livee.org/WXT510/03000005c3a2/" latitude="35.712194" location="東大情報基盤センター" longitude="139.76775" sensorModel="WXT510" sensorVendor="vaisala" >

<sensor id="live-e.org/WXT510/03000005c3a2/Temperature" sensorType="Temperature"> $<$ value time="2006-07-25T19:37:33.0000000+09:00" $>25.8</$ value $>$

$<$ /sensor $>$

<sensor id="live-e.org/WXT510/03000005c3a2/Humidity" sensorType="Humidity" > $<$ value time="2006-07-25T19:37:33.0000000+09:00">73.3</value $>$

$<$ /sensor $>$

<sensor id="live-e.org/WXT510/03000005c3a2/Pressure" sensorType="Pressure"> $<$ value time="2006-07-25T19:37:33.0000000+09:00">1009.4</value $>$

$<$ /sensor $>$

<sensor id="live-e.org/WXT510/03000005c3a2/RainFall" sensorType="RainFall"> $<$ value time="2006-07-25T19:37:33.0000000+09:00" $>0</$ value $>$

$<$ /sensor $>$

<sensor id="live-e.org/WXT510/03000005c3a2/DayRainFall" sensorType="DayRainFall"> $<$ value time="2006-07-25T19:37:33.0000000+09:00" >0.64</value $>$

$<$ /sensor $>$

<sensor id="live-e.org/WXT510/03000005c3a2/WindDir" sensorType="WindDir">

$<$ value time="2006-07-25T19:37:33.0000000+09:00" >174</value $>$

$<$ /sensor $>$

<sensor id="live-e.org/WXT510/03000005c3a2/WindSpeed" sensorType="WindSpeed"> $<$ value time="2006-07-25T19:37:33.0000000+09:00">0.9</value $>$

$<$ /sensor $>$

$</$ sensorGroup $>$

Fig. 5. Example of reply as XML document

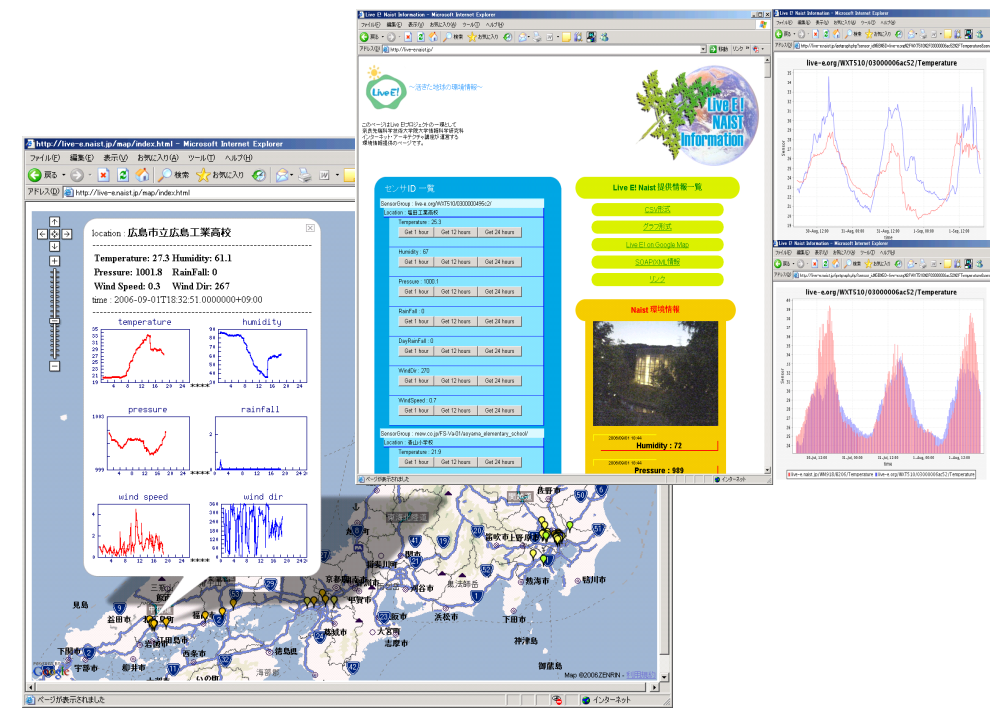

Fig. 6. web page providing weather information at each place 


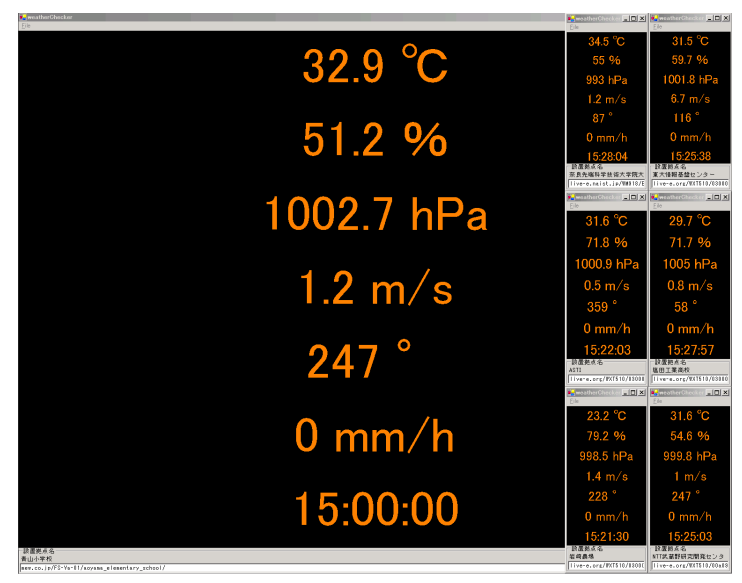

Fig. 7. weather information viewer

Figure 8 shows another application which displays weather information. This application shows the status of clouds and path information of a typhoon with sensor data. Live E! Project doesn't observe cloud and typhoon. Collaborating with other organizations, our project provides these data. Just one organization never collects all kinds of information. We enhance the collaboration with others.

As shown in figure 3, the sensors are connected to the IPv4 and IPv6 dualstack network with multicasting capability. The sensor information can be simultaneously deliver to multiple nodes, that want to get the sensor information, without requesting the copying data at the sensor node. Topping on the IPv4/IPv6 multicast-capable IP network, the Live E! overlay network is defined. The live E! overlay network has the distributed database system across the nation-wide Japan. This is for load balancing and for robust data management against some system failure. Also, as shown in the figure, this system has already included Web camera for obtaining the live video information and the sound information from the network.

We have installed more than 100 stations and let them on-line. Some of stations have installed in Philippine by the collaboration with ASTI [8, or have installed in Thailand by the collaboration with PSU 9 .

For dense installation of weather sensors, we collaborate with Minato-ku in metropolitan Tokyo. Several sensors are installed at some elementary schools at Minato-ku. We will install about 30 stations in this fiscal year of 2006. By this installation, Minato-ku can have about $2 \mathrm{~km}$ mesh weather station network.

\subsection{Future Enhancement of Internet Weather Station System}

- XML based Publish/Subscribe system

The more sensor devices and users increase, the more data and queries should be processed. The most popular web-service is "getCurrentDataAll()". Users are very interested in real time data. In order to provide this web-service 


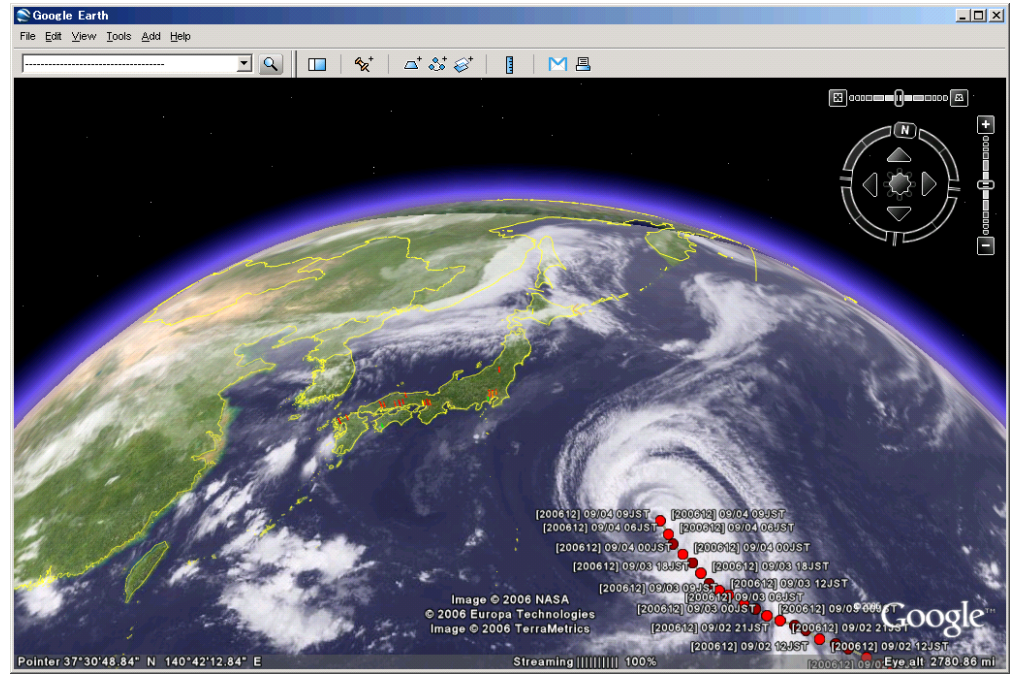

Fig. 8. data displaying on google earth

stable, we create XML based Publish-Subscribe system (Figure 9). This system don't store archived data but only provide current data. If users register for subscribing data, they constantly receive current data of all sensors.

- Integration with InternetCAR system

WIDE project has long time worked on the R\&D activity, where automobiles connect to the Internet. Automobiles can be realized as the mobile sensor node, running on the surface of the earth. We are now integrating the "InternetCAR" into our platform [10. In addition, we are trying to use ad-hoc network for sensors not having the connectivity to the internet. Patrol nodes (ex. Post office cars, garbage trucks or buses) gather the sensing data via ad-hoc network [1].

- Support small nodes

There are lots of microcomputers not having the ability to translate XML document. In order to integrate lots of data created by microcomputers generate, we study about XML translational machinery [12].

- Scalable and autonomous data collection and distribution system

The current system is a kind of client-server system. This is just fine for small scale system. However, the system must come up with the increase of sensors and the increase of type of sensors. Distributed and autonomous management and operation is mandatory. We are studying about geographical location based peer-to-peer network [13].

We are evaluating the requirements of future sensor network, and integrate several kinds of researches. 


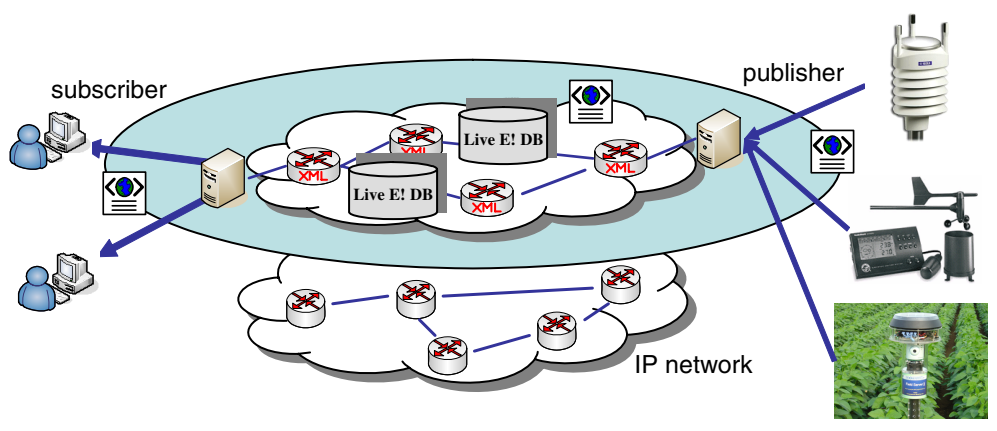

Fig. 9. XML based publish/subscribe system

\section{Conclusion}

The Live E! project is an open research consortium among industry and academia to explore the platform to share the digital information related with the earth and our living environment. Using the low cost weather sensor nodes with Internet connectivity, we deployed the nation-wide sensor network. The network has accommodate more than 100 station, and has two of dense installation. The application of this weather station network is for disaster protection/reduction/recovery and for educational material for various level of students.

\section{Acknowledgment}

The authors thanks to all the organization and peoples contributing to the Live E! project. These are; WIDE project sponsor organizations, NiCT/JGN, Netone Systems Co.Ltd, Echelon Japan KK, WeatherNews Inc., NTT Neo-mate, Wilcom Japan Ltd., IRI Ubiteq Inc., NTT East Corp., Matsushita Electric Works Ltd., Cisco Systems Japan K.K., Dai-Dan Co.Ltd., Uchida Yoko Co.Ltd., INTEC Inc., Shimizu Corp., Yamatake Corp., OBIS Corp., and Mitubishi Research Institute Inc.

\section{References}

1. Lawrence Lessig, "The future of Ideas; The Fate of the Commons in a Connected World" Random House Inc., ISBN 0375505784, November 2002.

2. Home Page of BACnet, http://www . bacnet.org/

3. Home Page of Lon Mark, http://www.lonmark.org/

4. Kyoto Protocol by United nation, http://www.cop3.org/

5. Home Page of Live E! Project, http://www.live-e.org/

6. Minato-ku, Tokyo, http://www.city.minato.tokyo.jp/

7. Kurashiki-City, http://www.city.kurashiki.okayama.jp/ 
8. ASTI (Advanced Science and Technology Institute), http://www.asti.dost.gov.ph/

9. PSU (Prince of Songkla University), http://www.psu.ac.th/

10. Shinichi Doui, Satoshi Matsuura, Hideki Sunahara: An Infrastructure for Environmental Information Gathered by Fixed-point and Mobile Sensors, Multimedia, Distributed, Cooperative and Mobile Symposium(2006), IPSJ Symposium Series Vol.2006, No.6, $801-804$.

11. Hiroki Ishizuka, Kenji Sasaki, Satoshi Matsuura, Makoto Kamiya, Hideki Sunahara, Hiroshi Esaki: Collecting Adaptive Data for Isolated Wireless Sensors with Patrol Nodes in Live E!, International Workshop on Future Mobile and Ubiquitous Information Technologies, Proceeding of FMUIT2006, 249 - 253.

12. Hideya Ochiai, Hiroshi Esaki: The Application of XML Translator for a Large-Scale Sensor Node Network, IEICE 2006, A-21-5.

13. MATSUURA Satoshi and FUJIKAWA Kazutoshi and SUNAHARA Hideki: Mill: Scalable Area Management for P2P Network based on Geographical Location, Proceedings Euromedia 2006, $46-52$. 